\title{
Power-Dependable Transactions in Mobile Networks
}

\author{
Ami Marowka \\ Software Engineering Department \\ Shenkar College of Engineering and \\ Design \\ 12 Anna Frank, Ramat-Gan, 52526, \\ Israel \\ E-mail: amimar2@yahoo.com
}

\author{
David Semé \\ LaRIA : Laboratoire de Recherche en \\ Informatique d'Amiens \\ Université de Picardie Jules Verne \\ 33 rue Saint Leu, 80000 Amiens, \\ France \\ E-mail: David.Seme@u-picardie.fr
}

\begin{abstract}
We define a Quality-of-Power-Service (QoPS) metric to evaluate the efficiency of power-aware routing protocols in wireless ad-hoc networks. The aim of power management of routing protocols is to prolong the life-time of individual nodes in wireless network and thus to increase the delivery rate of Unicast transactions.

QoPS metric is applied to different location-based Unicast transaction protocols. The results confirm that powerrelative distribution of data streams in multi-paths Unicast transaction protocols consume substantially less energy from individual nodes than from other distribution methods. The locality distribution phenomenon discovered by the simulations explains, on the one hand, the long lifetime of large, dense, and highly degree wireless networks, and on the other hand, the short lifetime of small, sparse, and low degree networks
\end{abstract}

\section{Introduction}

The convergence of computing and communications is driving the technology industry to design mobilized solutions. The mobile era is also the power-aware era [1]. Staying within the microprocessor power envelope becomes a major design obstacle in the design of future high-end microprocessors. Increasing the performance of a microprocessor by $1 \%$ costs $3 \%$ growth in power consumption. Thus, the main challenge is not to break the "power wall." Handling and managing power correctly becomes a critical feature for software targeted for mobile computing.

Power management in wireless networks is an essential factor for their smooth function. Wireless nodes, especially sensors, use small batteries for energy supplies that in many cases cannot be replaced. Therefore, energy conservation is a vital factor in a sustained network lifetime. It was shown in [2] that wireless nodes often expend most of their energy in communications. As such, the objective is to minimize the aggregate transmission power (energy) used by individual nodes to route data along multiple paths.

Performance evaluation of power-aware protocols commonly use three metrics: minimum-energy routing, max-min routing, and minimum-cost routing [11]. Minimum-energy routing minimizes the total energy consumed as a packet is forwarded on a route. This routing does not take into account the remaining battery power and thus does not evaluate the network lifetime. The max-min metric chooses the route that maximizes the minimum residual energy of any node on the route. Routes that are chosen by this mechanism tend to be longer and have greater total energy consumption than minimum-energy routing. Minimum-cost routing minimizes a cost function that takes into account both the remaining battery power and the transmission energy of neighboring nodes. However, these 
metrics select the optimal route and do not provide maximum-lifetime routing.

The lack of appropriate definition and metric for evaluating the lifetime of an ad hoc wireless network drives the research in [12].This is the first paper, to the best of our knowledge, that defines the network's lifetime accurately and establishes a comprehensive algorithmic methodology for the maximization of lifetime in wireless networks. However, this is an important theoretical research based on centralized solutions where for practical use distributed solutions are more appropriate.

The contribution of this work is twofold. First, we define a new metric called Quality of Power Service (QoPS) for evaluating the lifetime of an ad hoc wireless network. QoPS extends the definitions in [12] to the real world. That is, QoPS enables us to evaluate, in quantitative manner, the energy consumption of distributed protocols. It also allows us to capture practical issues which absent from global and theoretical solutions such as taking in account not only the transmission power consumption but also the power consumption during receiving and idle times. Moreover, practical evaluation of distributed protocols should take in account energy losses due to re-transmissions and reroutings caused by power failures of nodes.

The second contribution is developing and evaluates four Unicast transaction protocols. They have common properties: all are location-based protocols, scalable, localized, and use progression algorithms. The progression manner of the algorithms enable them to be loop-free, while the use of multi-paths enables high delivery rate transactions of high energy efficiency. The evaluation of the protocols is done by using the new QoPS metric.

The tested Unicast protocols help us to address the problem of how to deliver a large data stream from an origin node to a destination node in an ad hoc wireless network. The goal is to find the best routing protocol that enables the execution a maximum number of random and simultaneous independent transactions over the network without a power failure. An ad hoc network is a self-organized mobile network, where every node is responsible for the computation and the communication operations. In this paper we are primarily concerned with ad hoc networks that have relatively static topology, common examples of which are sensor networks.

The remainder of the paper is as follows: Section 2 defines a power-aware network model. Section 3 defines the QoPS metric. Section 4 presents the Unicast protocols that are used throughout the paper. Section 5 describes the simulator and the simulations. This is followed by results, detail analysis, including performance comparisons between the tested protocols. Section 6 ends the paper with a few conclusions. The related-works section is not presented in this version due to space limits. However, their references appear in the references section.

\section{A Power-Aware Network Model}

In this section, we formally define a simplified but widely accepted model of a power-aware wireless ad-hoc network that will be used later in our simulator and simulations. A realistic model should take in account many parameters such as mobility of the nodes and the fact that radio coverage area is extremely irregular and influenced by weather conditions and by interference with preexisting infrastructure (e.g., power lines, base stations, etc.) However, including these details in the network model would make it extremely complicated and scenariodependent, hampering the derivation of meaningful and sufficiently general analytical results. For this reason, the model we define here makes a few assumptions that make it simple.

We formally define a power-aware network model as follows: 


\section{Definition 1: A power-aware network model}

A power-aware network model is an undirected graph

$G \equiv(V, E, b, t, r, i, s)$, where:

1. Vis the set of nodes;

2. $E$ in $V \times V$ is the set of undirected edges, i.e., $(u, v)$ in $E$ if $u$ is able to transmit to $v$;

3. $D$ an average node degree;

4. For $v$ in $V, b(v)$ is the initial energy

("battery") of node $v, t(v), r(v), i(v)$ and $s(v)$ are the power required for $v$ to transmit, receive, to be in idle mode and to be in sleep mode respectively.

Node $i$ in $V$ is said to be stationary if its physical placement does not vary with time. If all the nodes are stationary, the network is said to be stationary. To simplify the metric definitions and the simulations we assume that the network is stationary.

The nodes communicate using omnidirectional antennas, which means that when a node is using enough power to transmit over a distance $d$ the transmission can be received by any other node that is not distant from the sender by more than $d$. Moreover, we assume that all the nodes are equipped with transceivers having the same features. This means that the network is a homogeneous network consisting of nodes which have the same transmission range, the same initial battery power and the same transmission, receiving and idle power consumption. The common transmission range induces a strongly connected communication graph. This graph is often called the unit disk graph model in the routing protocols literature.

We assume that the routing sessions are issued in a random manner. Therefore, the origin node and the destination node of each routing are chosen randomly and there is no a-priori knowledge available regarding the future sessions.
The routing requests in our model are managed by localized location-based routing protocols [6]. In such protocols, the location of destination node is known and the distance between neighboring nodes can be estimated on the basis of incoming signal strengths. The routes between nodes are created through several hops in localized manner where each node makes decision to which neighbor to forward the message based on its own location, its neighboring nodes, and the destination.

\section{Quality of Power Service}

In the fields of packet-switched networks and computer networking, the traffic engineering term Quality-of-Service (QoS) refers to the probability of the telecommunication network meeting a given traffic contract or, in many cases, is used informally to refer to the probability of a packet succeeding in passing between two points in the network.

There are essentially two ways to provide QoS guarantees. The first is simply to provide lots of resources, enough to meet the expected peak demand with a substantial safety margin. This is nice and simple, but some people believe it to be expensive in practice, and unable to cope if the peak demands increases faster than predicted because deploying the extra resources takes time. Ad hoc wireless networks usually provide lots of resources for free such as multi-paths between each pair of nodes. Our protocols use this redundancy to decrease the possibility of problems due to energy losses.

The second option is to require people to make reservations, and to only accept the reservations if the routers are able to serve them reliably. In ad hoc wireless networks terminology it means that each locationbased transmission from a source node to a destination node is done in two phases. In the first phase, the routing discovery phase, the protocol searches for paths that meet the transmission restrictions. The second phase is the actual data delivery. 
Ad hoc networks call for an extension of the traditional definition of the term QoS. We define a new term, Quality-of-Powerservice (QoPS), which refers to the probability of a data transaction succeeding in passing between two points in ad hoc wireless network without a failure caused by loss of power.

Before we formally define the QoPS metric the basic communication operation that will serve us in the simulations is defined.

\section{Definition 2: Unicast Transaction}

A Unicast transaction is an atomic unit of data delivery between two nodes in ad hoc wireless network.

A simple transaction usually uses a few paths to accomplish the data transmission. If the transaction fails on any path, the protocol tries to retransmit the data by using the other paths.

A single transaction might require two phases: routing discovery and data delivery. When this happens it is usually important to be sure that the transaction is not left with only one of the phases carried out. For a transaction to be completed, a transaction has to be completed in its entirety. Also, transactions should not interfere with each other.

\section{Definition 3: Quality of Power Service (Network's Lifetime)}

Let $N$ be a power-aware network model and $P$ a routing protocol. The Quality-ofPower-Service (QoPS) of $P$ over $N$ is the number of transactions that are performed continuously without a power failure.

Quality-of-Power-Service (QoPS) measures the network's lifetime. It is a quantitative metric that evaluates the ability of an application to obtain the network service it requires for successful operation as a function of the aggregate battery energy of the network's nodes.
In the next section we describe our poweraware Unicast protocols and use the QoPS metric to evaluate their performance and to compare them.

\section{Power-Aware Unicast Protocols}

In this section we describe four Unicast protocols for delivering large streams of data from an origin node to a destination node. Each protocol tries to minimize the power consumption of the transition nodes that participate in the transaction in a different manner, but all four have a common purpose: to prevent failure of nodes due to an energy loss that might detach the fragile connectivity of the wireless network.

We start by describing the common design characteristics of the protocols based on various classifications used in the open literature.

Location-Based Protocols. In positionbased protocols the routing is performed by a scheme that is based on the geographical location of the nodes. The distance between neighboring nodes can be estimated on the basis of incoming signal strengths and mutual exchanging of information between neighbors. During initialization, each node gathers information about its neighbors, including each neighbor's position, direction, remaining battery power, and the total number of neighbors. This information can be provided by the Global Positioning System (GPS). Next, the node forwards the data to the chosen neighboring node based on the protocol decision policy.

Localized Protocols. Localized protocols are distributed and scalable mutual communication schemes, where a local decision achieves a desired global objective. In localized protocols, each node makes a decision as to which neighbor to forward the message based only on the location of itself, its neighboring nodes, and the destination.

Progression Protocols. In a progression protocol, a node $A$ chooses its neighbors for forwarding the data based on 
geographic distance. Only neighboring nodes that are closer to the destination than node $A$ are considered [7]. In our protocols, a node distributes its data among all his neighboring nodes that show progression. Let $A, B$ and $C$ be an origin node, an intermediate node, and a destination node respectively.

Node $B$ is a progression node iff $|B C|<$ $|A C|$.

Our basic concept for increasing the power-lifetime of a network is by minimizing the energy consumption of individual nodes by distributing the transmission data among its neighboring nodes. However, preliminary experiments show that it's more efficient to distribute the data stream among the neighboring progression nodes rather than among all neighboring nodes.

Loop-Free Protocols. It was shown that progression protocols such as GEDIR and MFR [7] are loop-free protocols. However, due to the mobile nature of adhoc wireless networks that causes frequent unpredictable position changes, our protocols cannot be classified as deliveryguaranty protocols. Nevertheless, as our simulations show, we achieve high delivery rates by virtue of using multipaths.

One-Phase Protocols. Usually, ondemand routing is composed of two phases: route discovery and data delivery. In route discovery, a source uses flooding to acquire a route to its destination. This degrades system-wide energy conservation. The transit nodes, upon receiving a query, learn the path to the source and enter the route in their forwarding tables. The destination node responds using the path traversed by the query. Thus, in on-demand protocols, route discovery and data delivery may become inefficient and therefore the lifetime of the wireless nodes decreases. Consequently, we design our Unicast protocols as one-phase protocols where the discovery phase and the data delivery are performed at the same phase.
Traceless Protocols. Traceless protocols are localized schemes where nodes do not need to memorize past traffic and do not use routing-tables. All routing decisions are based on the location of neighboring nodes, the location of the destination, the position of the neighboring node that forwarded the message in the previous step, and the information that arrives with the message.

Self-Recovery Protocols. A power-aware Unicast transaction uses all the possible progression paths for delivering the stream of data from an origin node to a destination node. The redundancy of a multi-paths transaction reduces the possibility of a delivery failure. If one of the paths fails to forward a message to the destination, the protocol tries to retransmit the data by using another path. Only failure of all the paths will cause the failure of the entire transaction. The multipaths and multi-hops nature of wireless networks not only increases resiliency against power loss and mobility of the nodes in the network, but also increases the delivery rate of transactions.

Now, we are ready to present four Unicast protocol schemes. The protocols differ in the way they distribute the transmission packages among the nodes. All of them are location-based, localized, progression, loop-free, one-phase, traceless, and selfrecovery protocols as described above.

Even Distribution Protocol (EDP). In EDP protocol the transmission of a stream of data from a node to its progression neighbors is done by distributing the stream evenly among them. If the amount of data a node holds is less than a small threshold, then the data is forwarded to one of the neighboring nodes.

\section{Random-Distribution Protocol (RDP).} In RDP protocol the transmission of the data stream from a node to its neighbors is done by distributing the stream randomly among them. If the amount of data that a node holds is less than a small arbitrary threshold, then the data is forwarded to one of the neighboring nodes. 
Power-Distribution Protocol (PDP). In PDP protocol the transmission of the data stream from a node to its neighbors is done by distributing the stream relative to their remaining energy. For example, if the stream size is 100 Bytes, and there are two neighboring nodes with 25 and 75 units of energy respectively, then the data distribution will be 25 Bytes and 75 Bytes respectively. In other words, the "rich" node will contribute more energy.

In the above protocols, if the amount of the data that a node holds is less than a small arbitrary threshold, then the data is forwarded to one of the neighbor nodes. The neighbor nodes that participate in the transaction are those that show an ongoing progression toward the destination. If one of the nodes is unable to host its piece of data because it has not enough power to forward the data along the path, then the protocol distributes the data among the rest of neighboring nodes. If none of the neighboring nodes has the required power, the transaction fails.

Shortest-Path Protocol (SPP). In SPP protocol the transmission of stream data from a node to its neighbors is done by choosing the shortest-path between them. The shortest-path is referred to the sourcedestination shortest-path among the subset of ongoing progression paths between the source node and destination node (a local shortest-path), and not to the optimal shortest-path (a global shortest-path).

\section{Simulator and Simulations}

Experiments were carried out using a special-purpose ad-hoc network. We generated a database of 200 random unitgraph networks of various numbers of nodes and various average-node-degrees to get sparse and dense networks. Table 1 shows only a representative sample of many more simulations.

The network generator partitions the plane into rings of width $\mathrm{R}$, where $\mathrm{R}$ is the transmission radius. The number of nodes in each ring is determined relative to the number of nodes in the inner ring. If $n$ nodes are randomly located in the inner ring of $k$-rings network, then $n^{*} k^{2}$ nodes are randomly located for the entire network. The networks are generated incrementally, ring after ring. Each $k$-rings network is an extension of a (k-1)-rings network. In this way we can check exactly the scalability behavior of our protocols.

Table 1 is a comparison of the four protocols EDP, RDP, SPP, and PDP. Each comparison is done for all four protocols for the same network size and the same source-destination route. At the beginning of each benchmark, each battery's node is charged with $\mathrm{E}$ units of energy. In each transaction a package of $\mathrm{P}$ data-size is delivered from an origin node to a destination node. In our simulations we use $\mathrm{P}=100$ for two cases of energycharges: $E=1000$ and $E=10000$. The power consumption of a node is calculated relative to the amount of data passed. We adopted the power consumption of IEEE 802.11 interface as appeared in [11] where transmit consume 1.4-1.9 $\mathrm{W}$, receive 1.3$1.4 \mathrm{~W}$, idle mode $1.34 \mathrm{~W}$ and sleep mode $0.075 \mathrm{~W}$. In our simulations, transmission of one unit of data consumes one unit of energy and receives 0.7 unit of energy. We assume that the nodes are in sleep mode and are awakened only for receiving and transmitting of data. We ignore the energy consumption during sleep mode.

Analysis of the results shown in Table 1 discovers the following findings.

First, the PDP protocol outperforms all the other protocols. The results confirm our assumption that a power-aware protocol has to take into account the status of the remaining battery charge as the primary decision parameter. The EDP protocol comes next and shows good performance while supporting our basic energy-saving concept: maximum data-distribution maximizes the power-saving of an individual node and thus maximizes the lifetime of the entire network. The performance of the RDP and SPP protocols does not show any preference of one over the other. 


\begin{tabular}{|c|c|c|c|c|c|c|}
\hline \multicolumn{7}{|c|}{ Q QUALITY-OF-POWER-SERVICE } \\
\hline & \multicolumn{3}{|c|}{$\begin{array}{c}\text { Battery Charge } \\
=1000 \\
\text { Package-size }= \\
=100\end{array}$} & \multicolumn{3}{|c|}{$\begin{array}{c}\text { Battery Charge } \\
=10000 \\
\text { Package-size }= \\
100\end{array}$} \\
\hline \multirow[t]{2}{*}{ Nodes } & \multicolumn{3}{|c|}{$\begin{array}{l}\text { Average } \\
\text { Degree }\end{array}$} & \multicolumn{3}{|c|}{$\begin{array}{l}\text { Average } \\
\text { Degree }\end{array}$} \\
\hline & 4 & 7 & 9 & 4 & 7 & 9 \\
\hline 27 & & & & & & \\
\hline & 38 & 47 & 74 & 241 & 247 & 254 \\
\hline RDP & 33 & 4 & 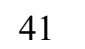 & 36 & 240 & 246 \\
\hline SPP & 36 & 3 & & 38 & 240 & 245 \\
\hline $\mathrm{PD}$ & 12 & 5 & & 254 & 260 & 269 \\
\hline Delivery & 94 & 9 & 96 & 91 & 92 & 92 \\
\hline 48 & & & & & & \\
\hline EDP & $T$ & - & 70 & 430 & 470 & 501 \\
\hline RDP & & 38 & & 405 & 420 & 450 \\
\hline SPP & 3 & 40 & & 337 & 421 & 444 \\
\hline PD & 4 & 59 & 10 & 486 & 498 & 567 \\
\hline Delivery & 92 & 94 & 96 & 90 & 92 & 94 \\
\hline 75 & & & & & & \\
\hline & 45 & 5 & & 565 & 601 & 656 \\
\hline & 3 & 4 & & 412 & 470 & 477 \\
\hline & 3 & 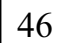 & & 472 & 420 & 432 \\
\hline & 50 & 5 & 1 & 682 & 712 & 750 \\
\hline Delivery & 90 & 94 & 97 & 88 & 92 & 95 \\
\hline
\end{tabular}

Table 1. QoPS of four protocols: EDP, RDP, SPP and PDP. Experiments were carried on networks of 27,48 and 75 nodes and average-degrees of 4, 7 and 9, for package-size of 100 and two batterycharges of 1000 and 10000 . Delivery is the average delivery rate in percents.

Second, using multi-paths routing for each transaction leads to a high delivery rate (marked Delivery in Table 1). The delivery rate increases with the increase in the average-degrees, as can be expected. However, when the battery charge is increased from 1000 to 10000 and the package-size remains 100 , the number of the transactions increases while the delivery rate is decreased. Moreover, the performance increases with the increase in the average-degrees because the opportunity to distribute the data among more neighboring nodes results in decreasing the power consumption of individual nodes.
Third, most of the energy consumption during a transaction is carried by a few neighboring nodes in the area around the origin node. Let's use an example to explain this phenomenon and its implications. Suppose that the subnetwork induces by an origin node and the nodes along the progression paths toward the destination node are an approximation of a binary tree where the origin node is the root. Thus, if the data distribution is done evenly among the neighboring nodes and the data-size is $\mathrm{P}$ units, then after $\log P$ steps of divide-and-distribute we are left with data units of size 1 . Thus, from here on the data packages are forwarded toward the destination without being divided anymore. Therefore, the nodes in a radius of $\log P$ hops from the origin node carry most of the energy consumption. Nodes closer to the origin node consume more energy then nodes farther away.

Now, let's analyze the implications of this phenomenon in light of the measurements in Table 1 of the PDP protocol. For very large networks and high average-degrees, the lifetime is maximal because in each transaction a small number of nodes participate in the most power-carried. In each new transaction, a new source destination is chosen randomly. Therefore, the probability that the new source node is located in the neighboring area of the origin node of the previous transactions is decreasing as the network size and the average-degrees grow.

\section{Conclusions}

In this paper we define a new metric called Quality-of-Power-Service (QoPS) that measures the lifetime of an ad hoc network for a given routing protocol. Using QoPS we evaluate four power-aware routing protocols. The aim of these protocols is to deliver large data streams through progression multi-paths routes from an origin node to a destination node in an ad hoc wireless network.

The Power Distribution Protocol (PDP) has shown the best ability to increase the 
life-time of a wireless network that totally relies on nodes which are based on a limit battery power to function properly. The excellent performance of the PDP protocol is derived from taking into account the remaining battery power of neighboring nodes. However, the EDP protocol has shown that even without knowing such information, a good performance can be achieved if the data distribution spans as many nodes as possible

\section{References}

[1] U. Weiser, Future direction in Microprocessors, Intel 'technology in motion' conference, May 30, 2005, RamatGan, Israel.

[2] L. M. Feeney, M. Nilson, Investigating the Energy Consumption of a Wireless Network Interface in an Ad Hoc Networking Environment, Proc. 20th IEEE INFOCOM, pp. 15481557,2001

[3] C. Siva, R. Murthy and B. S Manoj, Ad Hoc Networks, Architecture and Protocols, Prentice Hall, 2004

[4] S. Basgani, M. Conti, S. Giordano and I. Stojmenoviv, Mobile Ad Hoc Networking, IEEE Press, 2004.

[6] I. Stojmenovic, Position-Based Routing in Ad Hoc Networks, IEEE Communications Magazine, July 2002

[7] I. Stojmenovic and X. Lin, Loop-free hybrid single-path/flooding routing algorithms with guaranteed delivery for wireless networks, IEEE Transactions on Parallel and Distributed Systems, Vol. 12, No. 10, October 2001, 10231032.

[8] A. Srinivas and E. Modiano, Minimum Energy Disjoint Path Routing in Wireless Ad-hoc Networks, Mobile computing and networking, San Diego, CA, USA, Sep 14-19, 2003, 122-133, 2003.
[9] I. Stojmenovic and X. Lin, Power-aware Localized routing in Ad-Hoc Networks, IEEE Transaction on Parallel and Distributed Systems, Volume 12, Number 10, October 2001.

[10] J. Li and D. Cordes, Hybrid Greedy/multicasting Power-aware Routing Protocol in Ad Hoc Networks, Proceedings of the International Conference on Information Technology: Coding and Computing (ITCC'04), Apr 5-7 2004, 725, 2004.

[11] L. M. Feeney, Energy-Efficient Communication in Ad-hoc Wireless Networks, In Mobile Ad Hoc Networking, pp. 301-327, Editors S. Basagni, M. Conti, S. Giordano and I. Stojmenovic, IEEEWiley, 2004

[12] A. Orda and B. A. Yassour, Maximum-Lifetime Routing Algorithms for Networks with Omnidirectional and Directional Antennas, Proceeding of ACM MobiHoc'05, May 25-27, 2005, Urbana-Champaign, Illinois, USA, pp. 426-437.

[13] A. Safwat, H. Hassanein and H. Mouftah, Energy-Aware Routing in MANETs: Analysis and Enhancements, Proceeding of ACM MSWiM'02, September 28, 2002, Atlanta, Georgia, USA, pp. 46-53. 